\title{
Double-decker repair of partial anomalous pulmonary venous return into the superior vena cava
}

Hisayuki Hongu, MD, ${ }^{\mathrm{a}}$ Masaaki Yamagishi, MD, PhD, ${ }^{\mathrm{a}}$ Yoshinobu Maeda, MD, ${ }^{\mathrm{a}}$

Keiichi Itatani, MD, PhD, ${ }^{\mathrm{b}}$ Satoshi Asada, MD, ${ }^{\mathrm{a}}$ Shuhei Fujita, MD, ${ }^{\mathrm{a}}$ and Hitoshi Yaku, MD, PhD

\section{ABSTRACT}

Objective: Conventional procedures for partial anomalous pulmonary venous return (PAPVR) to the superior vena cava (SVC) still have serious complications, such as late SVC and/or pulmonary venous obstruction and supraventricular arrhythmia. We aimed to introduce our newly developed surgical technique with minimum right atriotomy and double-barreled arrangement of systemic and pulmonary venous channels (double-decker technique).

Methods: From 1998 to 2018, 21 consecutive patients with PAPVR to the SVC underwent this new procedure. The patients' median age and body weight were 4.4 years and $16.5 \mathrm{~kg}$, respectively. Two female patients underwent lateral thoracotomy. Postoperative hemodynamics of both venous channels were assessed using time-resolved 3-dimensional magnetic resonance phase contrast imaging in 6 patients.

Results: The median follow-up period was 11.0 years. There was no early mortality and late death. No patient required reoperation and/or intervention. All patients maintained normal sinus rhythm, and supraventricular arrhythmia did not occur. The median blood flow velocity of the neo-SVC and neopulmonary venous channel was 0.40 and $0.30 \mathrm{~m} / \mathrm{s}$, respectively. Using time-resolved 3-dimensional magnetic resonance phase contrast imaging, the straight and nonrestrictive flow and low wall shear stress were visualized in both venous channels.

Conclusions: Our newly developed double-decker technique is a useful alternative surgical procedure for PAPVR to the SVC. Late complications can be completely avoided using this method. Growth potential of both channels is also maintained. (J Thorac Cardiovasc Surg 2019;157:1970-7)

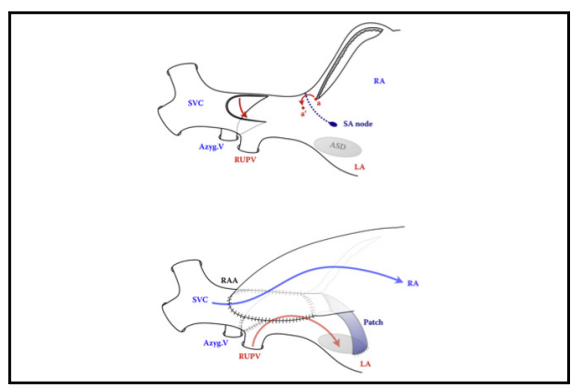

Double-decker technique for the partial anomalous venous return to the superior vena cava.

\section{Central Message}

The long-term outcomes of the double-decker technique appear clinically satisfactory. This method might be an alternative technique to the conventional procedures.

\section{Perspective}

The double-decker technique can avoid the late complications of conventional procedures, such as supraventricular arrythmia, and obstruction of the superior vena cava and/or pulmonary veins.

See Commentaries on pages 1978 and 1980.
The surgical procedure for partial anomalous pulmonary venous return (PAPVR) draining into the superior vena cava (SVC) is still controversial. Late SVC obstruction after end-to-end anastomosis between the SVC and right atrial appendage (RAA), supraventricular arrhythmia caused by over-long atriotomy, and pulmonary venous obstruction are serious complications of this. ${ }^{1-5}$ We developed and selected a new surgical technique with minimum right

From the a Department of Pediatric Cardiovascular Surgery, Children's Medical Center, and ${ }^{b}$ Department of Cardiovascular Surgery, Kyoto Prefectural University of Medicine, Kyoto, Japan.

Received for publication Oct 8, 2018; revisions received Dec 17, 2018; accepted for publication Jan 13, 2019; available ahead of print Feb 26, 2019.

Address for reprints: Masaaki Yamagishi, MD, PhD, 465 Kajii-cho, Kamigyo-ku, Kyoto, 602-8566, Japan (E-mail: myama@koto.kpu-m.ac.jp).

$0022-5223 / \$ 36.00$

Copyright (c) 2019 by The American Association for Thoracic Surgery

https://doi.org/10.1016/j.jtcvs.2019.01.057 atriotomy and double-barreled arrangement of systemic and pulmonary venous channels. ${ }^{6}$

Because of the flow complexity, the conventional examination methods, such as cardiac echocardiography velocity measurements or 2-dimensional flow magnetic resonance imaging, are limited in their ability. Time-resolved 3-dimensional magnetic resonance phase contrast imaging (4D-flow MRI) captures 3-directional velocity through the cardiac cycle; thus, it can measure the velocity of the blood flow in any direction, and functional evaluation of the

Scanning this $\mathrm{QR}$ code will take you to the article title page to access supplementary information.

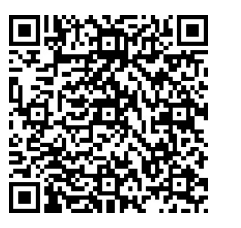




$\begin{array}{ll}\text { Abbreviations and Acronyms } \\ \text { ASD }= & \text { atrial septal defect } \\ \text { 4D-flow MRI = } & \text { time-resolved 3-dimensional } \\ & \text { magnetic resonance phase contrast } \\ & \text { imaging } \\ & =\text { left atrium } \\ \text { LA } & \text { magnetic resonance imaging } \\ \text { MRI } & =\text { partial anomalous pulmonary } \\ \text { PAPVR } & \text { venous return } \\ & =\text { pulmonary vein } \\ \text { PV } & =\text { right atrium } \\ \text { RA } & =\text { right atrial appendage } \\ \text { RAA } & =\text { superior vena cava } \\ \text { SVC } & \end{array}$

hemodynamics can be performed by calculating blood flow volume and measuring wall shear stress and the flow pattern. $^{7}$

\section{METHODS}

This study was approved by the institutional review board at Kyoto Prefectural University of Medicine (Date of approval: November 1, 2016; number: ERB-C-680-1). Clinical data were obtained from a retrospective review of the medical records as well as operative, echocardiographic, and catheterization reports. From November 1998 to August 2018, 21 consecutive patients with PAPVR to the SVC were identified, and no patient with PAPVR was excluded from the series. All 21 patients underwent the double-decker technique, and other procedures were not performed. Patients' median age at repair was 4.4 years (range, 0.955.9 years), and median body weight was $16.5 \mathrm{~kg}$ (range, 5.4-62 kg). Of the 21 patients, $8(38 \%)$ were male and $13(62 \%)$ were female. An atrial septal defect (ASD) was found in 14 patients $(67 \%)$; of these, sinus venosus defect was found in 7 patients $(50 \%)$, and patent foramen ovale in 7 patients $(50 \%)$. The other associated cardiac anomalies were as follows: persistent left SVC in 4 patients, tetralogy of Fallot in 2 patients, doubleoutlet right ventricle in 1 patient, cor triatriatum in 1 patient, ventricular septum defect in 2 patients, and aortic valve disease including truncus arteriosus in 2 patients. All but 2 patients underwent the operation through median sternotomy. In 2 female patients who had no associated cardiac anomalies without PAPVR, lateral thoracotomy was selected for esthetic purposes. Previous procedures were as follows: Blalock-Taussig shunt for pulmonary atresia in 1 patient, aortic valve replacement and Rastelli procedure for truncus arteriosus in 1 patient, and ASD closure in 1 patient. Postoperative hemodynamics of both venous channels were assessed using 4D-flow MRI in 6 patients with their approval.

The electrocardiogram was considered early if it was obtained before hospital discharge and late if obtained after discharge. Patients' electrocardiograms were interpreted as having a normal sinus rhythm, junctional rhythm, and supraventricular arrhythmias, including atrial flutter, atrial fibrillation, and multifocal atrial tachycardia.

\section{Operative Procedure}

Standard median sternotomy was the most common approach for simple PAPVR or PAPVR with only ASD cases, but minimally invasive approaches, including right thoracotomy, could be used as well. The SVC and the innominate vein were dissected free, and the azygos and anomalous pulmonary veins (PVs) were adequately exposed. Cardiopulmonary bypass
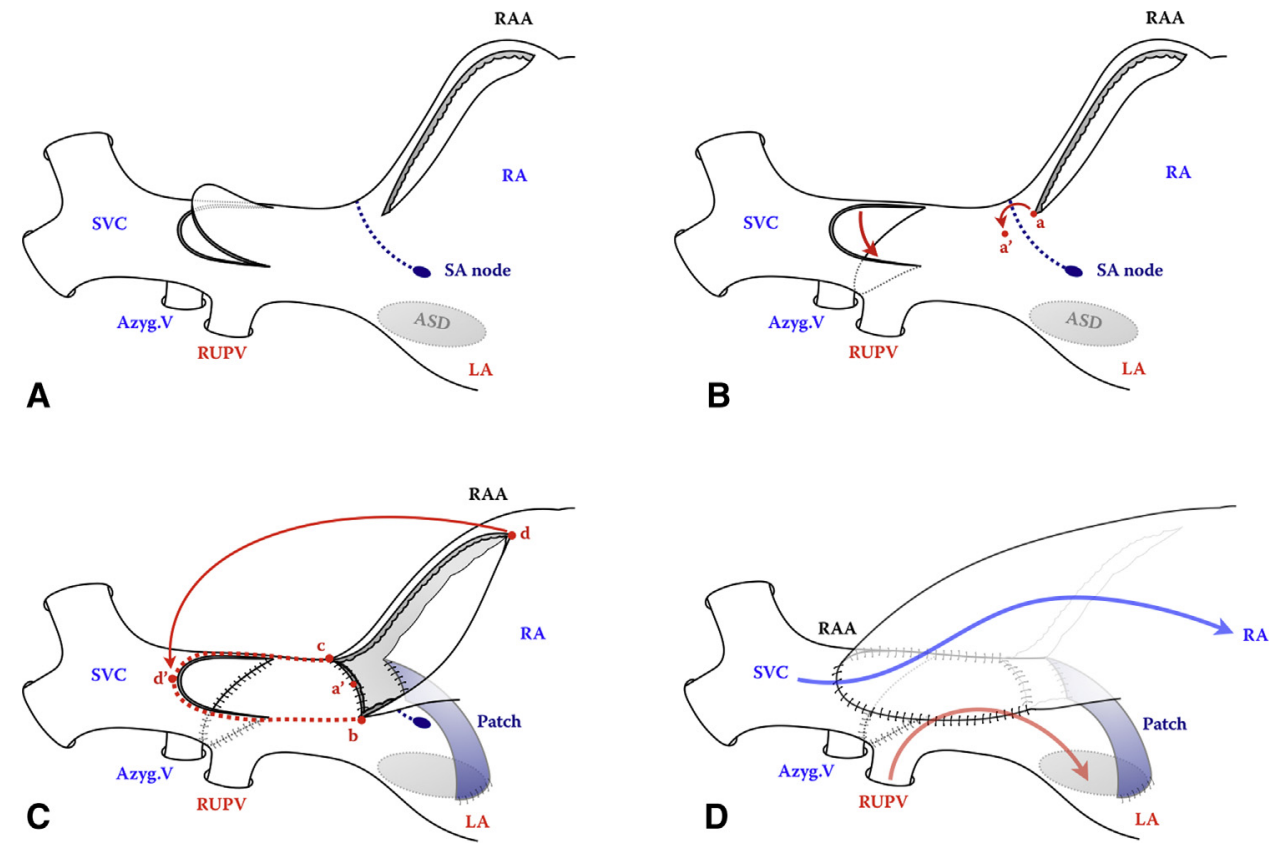

FIGURE 1. Double-decker technique; (A) the ridge line of the right atrium $(R A)$ between the right atrial appendage $(R A A)$ and the junction with the superior vena cava $(S V C)$ is incised. The ventral wall of the SVC is incised in an inversed U-shape to create the SVC flap. B, The SVC flap is translocated dorsally. The lower end of the right atrial orifice $(a)$ is anastomosed to the ventral external wall of the SVC $(a$ ') beyond the sinus nodal artery. C, Intra-atrial patch is anastomosed between the SVC orifice and the sinus venosus defect or patent foramen ovale $(A S D)$ to create a pulmonary venous blood pathway. The top of the right atrial incision $(d)$ is anastomosed to the distal edge of the SVC opening $\left(d^{\prime}\right)$. Points $b$ and $c$ are both end points of axial sutures to the superior vena cava from point $a$ ' and starting points to longitudinal suture for point $d^{\prime}$. Red dotted line indicates the suture line of the right atrial opening. D, Systemic and pulmonary venous channels are then created. SA, Sino-atrial; Azyg.V, azygos vein; RUPV, right upper pulmonary vein; $L A$, left atrium. 


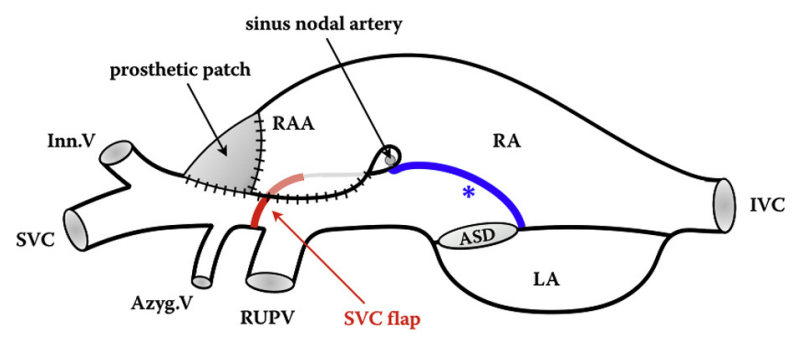

A

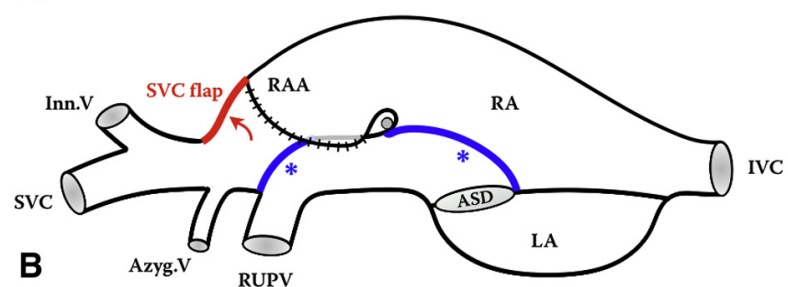

FIGURE 2. Modification of the double-decker technique; (A) distal end of the superior vena cava $(S V C)$ channel is supplemented by a prosthetic patch or an autologous pericardium. B, In the case of a small right atrial appendage $(R A A)$ flap and/or anomalous pulmonary venous drainage into the extremely cranial site of the SVC, the ventral wall of the SVC is incised in a U-shape to create the SVC flap (reversed in the original technique). In such a case, the ventral wall of the neo-SVC channel is reconstructed using the RAA flap and the U-shaped SVC flap; hence, the extension of the RAA flap to the SVC can be easily achieved compared with that in the original method. However, the border between the neo-SVC channel and the neopulmonary venous channel must be created using autologous pericardium, with no complications. *Indicates autologous pericardial patch. Inn.V, Innominate vein; $R A$; right atrium; $A S D$; sinus venosus defect or patent foramen ovale; $I V C$, inferior vena cava; $A z y g . V$, azygos vein; $R U P V$, right upper pulmonary vein; $L A$, left atrium.

was started using standard ascending aortic and bicaval cannulation. The SVC cannula was placed as high as possible in the SVC or in the innominate vein. A persistent left SVC was cannulated as an additional line.

After the cardiac chemical arrest, the ridge line of the right atrium (RA) was incised from the summit of the RAA toward the junction between the SVC and the RA (SVC-RA junction). The atriotomy was stooped a few millimeters short of the SVC-RA junction. The atriotomy was minimally extended to the caudal part from the summit of the RAA in some cases with large RAA (Figure 1, A). The sinus nodal artery (Figure 1, $A$, blue dotted line) was preserved. Just above the junction of the anomalous right upper PV, the ventral wall of the SVC was incised in an inversed U-shape to create the SVC flap (the SVC flap was translocated dorsally). The top of the flap was anchored just between the azygos vein orifice and the right upper pulmonary venous orifice. The flap was anastomosed to the inner wall of the SVC. The lower end of the right atrial orifice (Figure 1, $B$, point a) was anastomosed to the external ventral wall of the SVC (Figure 1, $B$, a') beyond the sinus nodal artery. A tiny longitudinal incision at the cephalic border of the SVC opening was added to enlarge the opening in some cases.

A flat autologous pericardial patch and/or a transposed caudal intraatrial septum was anastomosed between the SVC orifice and the sinus venosus defect to create a pulmonary venous blood pathway (Figure 1, C). In patients with an intact atrial septum, creation of a surgical ASD was necessary. The lower edge of the right atrial opening was widely anastomosed to the external wall of the SVC (Figure 1, $C, \mathrm{a}^{\prime}-\mathrm{b}, \mathrm{a}$ '-c). The top of the right atrial incision (Figure 1, C, d) was anastomosed to the distal edge of the SVC opening (Figure 1, $C$, d'). The side edge of the right atrial opening was anastomosed to the superior-lateral surface of the SVC external wall. The red dotted line indicates suture line of the right atrial opening (Figure 1, C, b-d', c-d').

Systemic and pulmonary venous channels were then created (Figure 1, $D$ ). Pulmonary venous blood drained into the left atrium (LA) through the proximal SVC, intra-atrial tunnel, and the sinus venosus defect. Systemic venous blood drained into the RA through the SVC ventral opening and the RAA baffle, which over-rode the proximal SVC. The proximal ventral wall of the SVC was shared as the dorsal wall of the systemic venous channel and ventral wall of the pulmonary venous channel.

Generally, the RA and RAA are large enough to reach the top of the RAA (Figure 1, $C$, d) to the cranial margin of the SVC opening (Figure 1, C, d') without any supplemental materials. However, supplemental material is exceptionally necessary in cases with a small RAA, especially in older patients and/or light right heart overload and/or anomalous PV drainage into the extremely cranial site of the SVC; for example, higher than the azygos vein. The gap between the cranial end of the RAA and the SVC opening is supplemented by a prosthetic patch or an autologous pericardium (Figure 2, A). Supplemental materials were used in new SVC channel reconstruction in 6 patients, including reoperation cases. Alternatively, the SVC flap was translocated ventrally. The SVC was partitioned just upstream of the pulmonary venous orifice using an autologous pericardial patch. The SVC channel was created by the ventrally uplifted SVC flap and the RAA (Figure 2, B) in 1 patient.
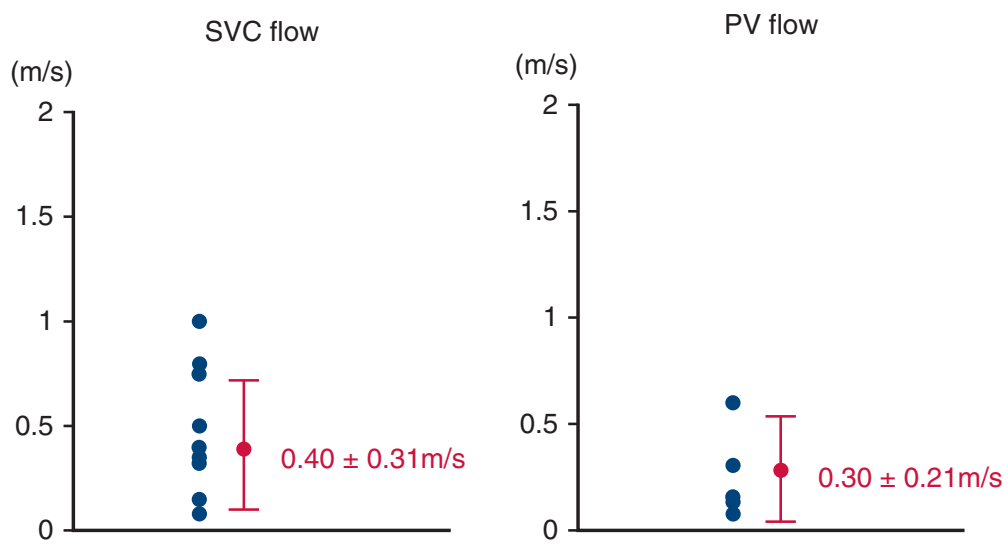

FIGURE 3. The flow velocity of both venous channels; both pathways show low flow velocity, which indicate no obstruction. $S V C$, Superior vena cava; $P V$, pulmonary vein. 


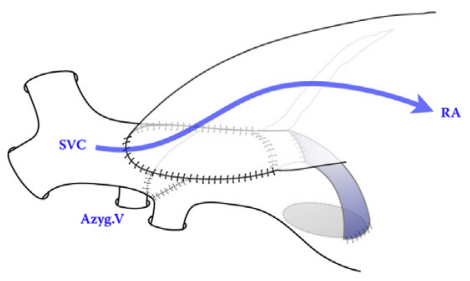

\section{flow streamline}

wall shear stress

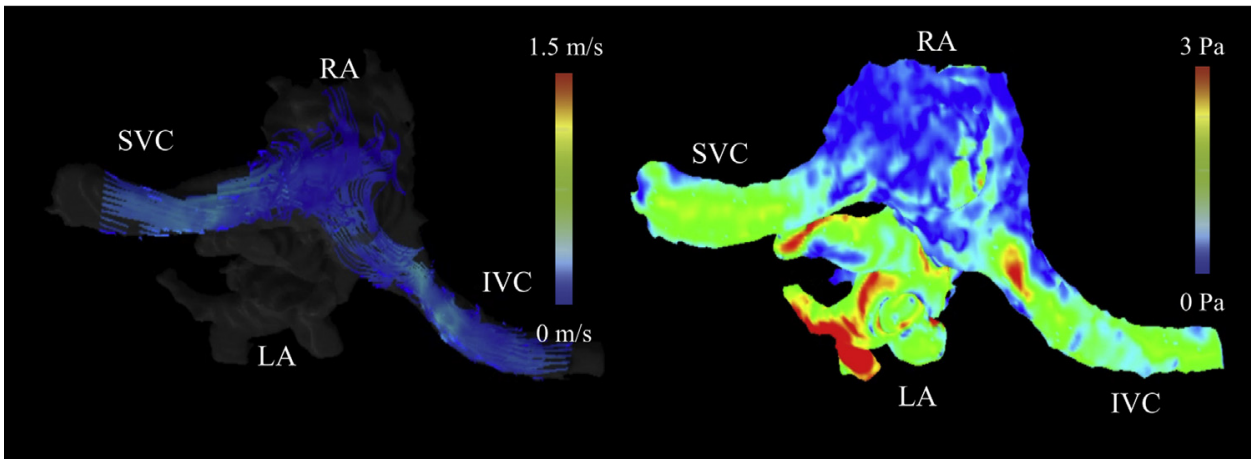

FIGURE 4. Time-resolved 3-D magnetic resonance phase contrast imaging analysis of the systemic venous channel; the superior vena cava ( $S V C)$ flow drains straight into the right atrium $(R A)$ without acceleration, and wall shear stress is very low. Azyg.V, Azygos vein; $I V C$, inferior vena cava; $L A$, left atrium.

\section{Statistical Analyses and 4D-Flow MRI Analyses}

Statistical analyses were performed using StatMate V, version 5.01 (Atoms, Tokyo, Japan). Regarding the magnetic resonance imaging (MRI) data acquisition, multislice sagittal steady state free procession image series were acquired in addition to 3-D cine phase contrast MRI, and 4D-flow MRI postprocessing was performed using iTFlow, version 5.1 (Cardio Flow Design Inc, Tokyo, Japan). Continuous data are presented as medians with ranges or as means with standard deviations.

\section{RESULTS}

The median follow-up period was 11.0 years (range, 1.718.7 years). Follow-up echocardiographic data and electrocardiograms were available for all 21 patients, and 4D-flow MRI for 6 patients $(29 \%)$. The median age of the patients with 4D-flow MRI during the operation was 2.4 years (range, 1.7-13.4 years). There was no early and late mortality. None of the patients required reoperation. All patients were categorized as class I in the New York Heart Association functional classification. No patient required medication therapy. As for arrhythmia, all patients preserved a normal sinus rhythm. Supraventricular arrhythmia did not occur in early or late stages. Besides, any type of arrythmia including transient junctional rhythm and nodal rhythm was not noted intraoperatively and/or postoperatively.

We measured the flow of neo-SVC and PV channels using cardiac echocardiography and 4D-flow MRI studies. The median flow velocity of neo-SVC and neo-PV channels was $0.40 \mathrm{~m} / \mathrm{s}$ (range, $0.08-1.00 \mathrm{~m} / \mathrm{s}$ ) and $0.30 \mathrm{~m} / \mathrm{s}$ (range, $0.08-0.60 \mathrm{~m} / \mathrm{s}$ ), respectively; they showed no PV and SVC obstruction (Figure 3). In the 6 patients who underwent 4D-flow MRI, the median flow velocity of neo-SVC and
neo-PV channels was $0.35 \mathrm{~m} / \mathrm{s}$ (range, $0.15-1.00 \mathrm{~m} / \mathrm{s}$ ) and $0.78 \mathrm{~m} / \mathrm{s}$ (range, $0.31-0.97 \mathrm{~m} / \mathrm{s}$ ), respectively. There was no significant difference between the 6 patients with $4 \mathrm{D}$ flow MRI and the others. In addition, flow streamline and wall shear stress of both venous pathways were visualized using 4D-flow MRI. The SVC flow drained straight into the RA without flow acceleration, and wall shear stress was almost less than $3 \mathrm{~Pa}$ at any point, which is sufficiently low in the pulmonary circulation system. The blood flow of the transitional zone between the distal SVC and the RAA flap was smooth and straight (Figure 4 and Video 1). Similarly, the straight flow of the neo-PV channel was clearly visualized, and wall shear stress showed a sufficiently low distribution. The wide pathway of the intra-atrial baffle

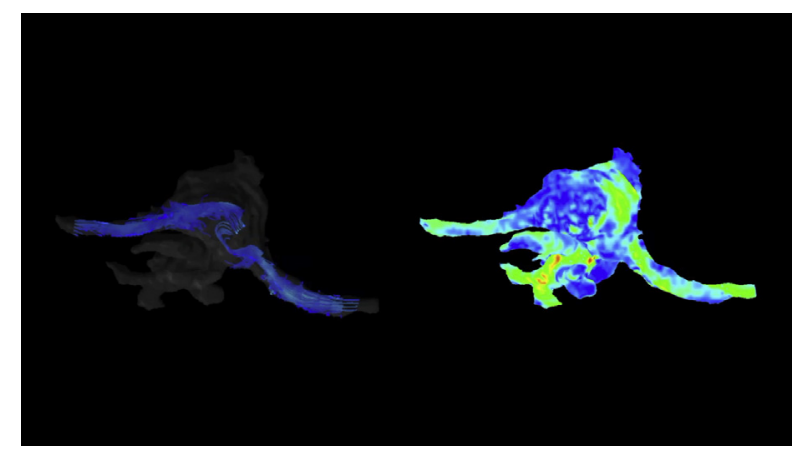

VIDEO 1. The blood flow of the transitional zone between the distal SVC and the RAA flap is smooth and straight. Video available at: https://www. jtcvs.org/article/S0022-5223(19)30268-5/fulltext. 


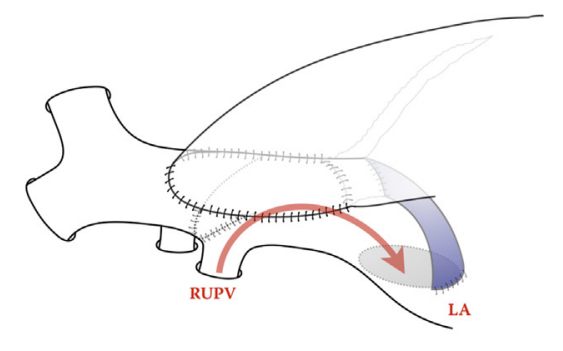

flow streamline

wall shear stress

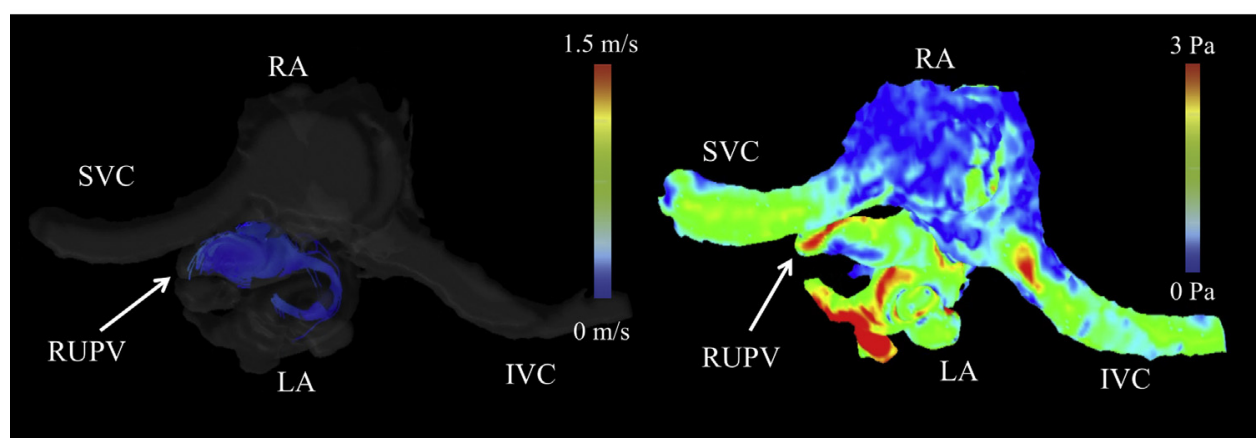

FIGURE 5. Time-resolved 3-D magnetic resonance phase contrast imaging analysis of the pulmonary venous channel; the neopulmonary pathway is visualized. The pulmonary flow drains straight into the left atrium $(L A)$ with no acceleration, and wall shear stress is very low. RUPV, Right upper pulmonary vein; $R A$, right atrium; $S V C$, superior vena cava; $I V C$, inferior vena cava.

was clearly shown (Figure 5 and Video 2). Double-decker flow was also clearly shown in the figure that was composed of the streamline of both venous pathways. The flow velocities of both pathways were almost the same (Figure 6 and Video 3).

\section{DISCUSSION}

PAPVR is a rare cardiac anomaly consisting of 1 or more, but not all, PVs connecting to the RA or systemic veins. According to the report of autopsy data, the prevalence of this condition is approximately 0.6 to 0.7 of the population. ${ }^{8}$ However, there are many undiagnosed PAPVR cases with no symptom; thus, the incidence actually might be greater. ${ }^{9}$

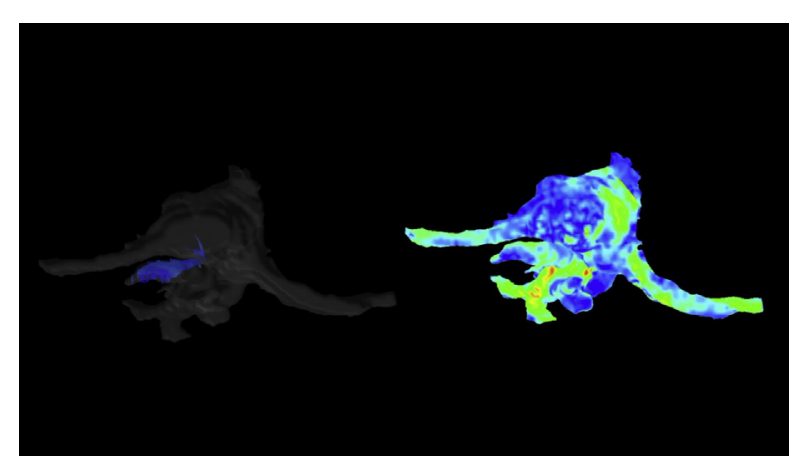

VIDEO 2. The wide pathway of the intra-atrial baffle is clearly shown. Video available at: https://www.jtcvs.org/article/S0022-5223(19)30268-5/ fulltext.
Sinus venosus defect, which is commonly located at the SVC-RA junction, accounts for 10 of all ASDs and $90 \%$ of all cases are associated with PAPVR. ${ }^{10,11}$ The aim of surgical treatment for PAPVR draining into the SVC is total correction with rerouting of anomalous PVs to the LA. Simple sinus venosus defect and/or patent foramen ovale closure or ligation of anomalous PV is not a curative operation. In addition, reimplantation of the anomalous veins to the LA generally was not performed because of its technical difficulty because of veno-atrial distance and long-term concern regarding veno-atrial anastomosis. $^{12}$ Moreover, for pediatric patients, avoiding anticoagulation therapy and maintaining growth potential are important; thus, artificial supplemental materials, including interposition of the vascular graft, should not be used as much as possible.

In terms of anatomic factors, the location of the sinus node, the sinus nodal artery, and the crista terminalis, the volume of the RA, and the diameter of the SVC complicate the procedures. ${ }^{13}$ There are many conventional procedures for PAPVR to the SVC. ${ }^{1-5,14-16}$ Their early outcomes are excellent overall, but they still have a few postoperative complications, such as supraventricular arrhythmias and venous obstruction, either systemic (involving mainly the SVC) or PVs.

In some conventional procedures, atriotomy is prolonged to make an autologous anterior atrial wall flap, which shares the venous channels. In addition, the atrial wall flap has to be anastomosed to the remaining atrial wall. The atrial 


\section{flow streamline}

composed flow

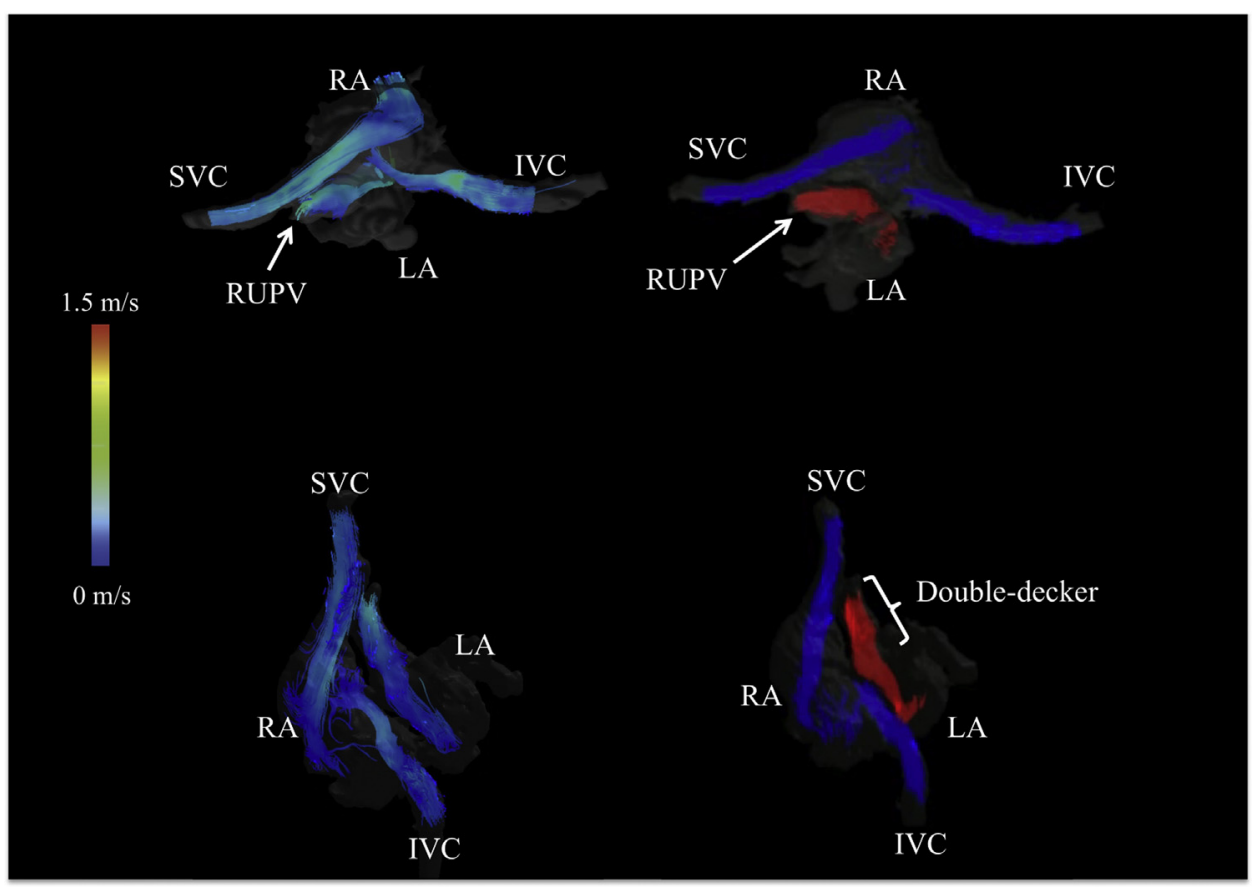

FIGURE 6. The composed streamline of both venous pathways. The red line shows the pulmonary venous return, whereas the blue line shows the systemic venous return. Clear double-decker flows are visualized. $R A$, Right atrium; $S V C$, superior vena cava; $I V C$, inferior vena cava; $R U P V$, right upper pulmonary vein; $L A$, left atrium.

wall flap size is anatomically limited; thus, the suture has a risk to run across the crista terminalis. In addition, in the case requiring enlargement of the SVC-RA junction to prevent the risk of SVC obstruction, the sinus nodal artery might be injured by the incision between the SVC and the RA. Postoperative supraventricular arrhythmias and venous obstruction along the suture lines are complications in such conventional procedures. Moreover, if the SVC is end-toend anastomosed to the RAA such as in the Warden technique, late SVC obstruction can occur, because there is a histological discontinuity. In the report by Said et al, in

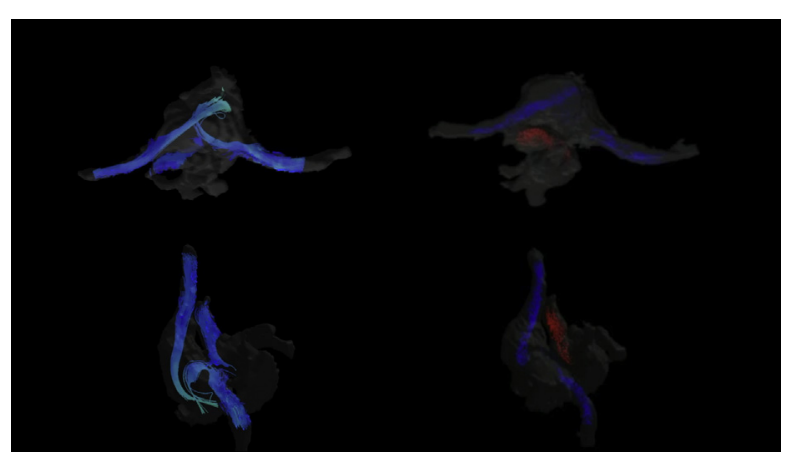

VIDEO 3. In this composed streamline, double-decker flows are clearly visualized. Video available at: https://www.jtcvs.org/article/S00225223(19)30268-5/fulltext. the single-patch method group, the risks of postoperative supraventricular arrhythmias, SVC obstruction, and PV obstruction were $8 \%, 5 \%$, and $5 \%$, respectively, whereas these were $12 \%, 4 \%$, and $12 \%$, respectively, in the double-patch method group. Moreover, in the Warden procedure group, these were $22 \%, 7 \%$, and 0 , respectively. ${ }^{17}$ The report showed that these problems were likely to occur in the conventional procedures, which is in contrast to the outcomes of our double-decker technique (Table 1).

We improved the innovative operative method called the double-decker technique for PAPVR to the SVC. ${ }^{6}$ The important point of this procedure is that the atriotomy is limited within a relatively safe zone from the sinus node, sinus nodal artery, and crista terminalis; thus, the risk of supraventricular arrhythmias is reduced. Moreover, the incisions across the cavo-atrial junction are unnecessary. In addition, this method can avoid the end-to-end anastomosis between the SVC and the RAA; then the risk of late stenosis of the systemic venous chamber can be decreased, because there is no histological discontinuity at the posterior wall of the systemic venous chamber. Additionally, in reconstruction of the PV channel, we only use an autologous tissue (autologous pericardium or atrial septal flap) without prosthetic materials; thus, the tissue's growth is expected, and the risk of reoperation is reduced. ${ }^{18,19}$ The proximal ventral wall of the SVC is shared as the dorsal wall of the systemic venous channel and as the ventral wall of the 
TABLE 1. Comparison of the risk of postoperative supraventricular arrhythmia, SVC obstruction, and PV obstruction in the conventional procedures and double-decker technique

\begin{tabular}{lccc}
\hline \multicolumn{1}{c}{ Procedure } & $\begin{array}{c}\text { Supraventricular } \\
\text { arrythmia }\end{array}$ & $\begin{array}{c}\text { SVC } \\
\text { obstruction }\end{array}$ & $\begin{array}{c}\text { PV } \\
\text { obstruction }\end{array}$ \\
\hline Warden technique & $22^{*}$ & $10^{*}$ & $0^{*}$ \\
Single-patch method & $8^{*}$ & $5^{*}$ & $5^{*}$ \\
Double-patch method & $12^{*}$ & $4^{*}$ & $12^{*}$ \\
Double-decker technique & 0 & 0 & 0 \\
\hline
\end{tabular}

Data are presented as percentages. $S V C$, Superior vena cava; $P V$, pulmonary venous. *Data from Said et al. ${ }^{17}$

pulmonary venous channel. The growth potential of both venous channels is effectively preserved. Moreover, because anastomosis of the entire circumference of the venous channels is unnecessary with this technique, future narrowing of both channels might be avoided. Furthermore, postoperative anticoagulation therapy was not needed. Median sternotomy and lateral thoracotomy are suitable in patients without other associated cardiac anomalies; thus, such patients who are particular with the appearance of the surgical wound are satisfied with this method.

The 4D-flow MRI is a novel blood flow imaging technology in vivo, ${ }^{20}$ which enables acquisition of time-resolved 3directional velocity data in the entire heart and/or all major thoracic vessels without radiation exposure. In conventional imaging examinations, such as cardiac computerized tomography or echocardiography, systematic evaluation of hemodynamics is not available. Computer flow simulation modeling on the basis of computational fluid dynamics is a useful technique to evaluate complicated hemodynamics similarly, but some of computational fluid dynamics calculations are on the basis of the assumption of boundary conditions, such as flow volume, outlet pressure, and chamber wall elasticity. In contrast, 4D-flow MRI is on the basis of the actual measured values. ${ }^{21-23}$ In the 4D-flow MRI analysis, not only blood flow drawn as the flow streamline (blood flow combined with flow velocity) but also wall shear stress can be measured. Wall shear stress is a stress on the endothelial wall caused by the near wall blood flow, and high wall shear stress is thought to be a predictor of intimal hyperplasia leading to vascular obstruction. Noninvasive 4D-flow MRI is extremely useful to rigorously analyze low pressure venous flow at sterically oriented venous channels.

\section{CONCLUSIONS}

The double-decker technique for PAPVR to the SVC showed excellent long-term results with excellent hemodynamics. Growth potential is effectively preserved. Late systemic and pulmonary venous channel obstruction and supraventricular arrhythmias are entirely avoided.
Moreover, the 4D-flow MRI analysis is a useful method to evaluate such complex configurations and hemodynamics.

\section{Conflict of Interest Statement}

Keiichi Itatani is an endowed chair of Kyoto Prefectural University of Medicine, financially supported by Medtronic Japan, has a stock option in Cardio Flow Design Inc, has received a KAKEN grant for young researchers, and is also a director of Hokkaido Cardiovascular Hospital. Masaaki Yamagishi is a consultant of W.L. Gore \& Associates, Inc. All other authors have nothing to disclose with regard to commercial support.

Image segmentation and postprocessing were performed by postgraduate students, Ms Hiroko Morichi and Ms Rina Makino.

\section{References}

1. Puig-Massana M, Murtra M, Revuelta JM. A new technique in the correction of partial anomalous pulmonary venous drainage. J Thorac Cardiovasc Surg. 1972; 64:108-13.

2. Chartrand C, Payot M, Davignon A, Guerin R, Stanley P, Bruneau J. A new surgical approach for correction of partial anomalous pulmonary venous drainage into the superior vena cava. J Thorac Cardiovasc Surg. 1976;71:29-34.

3. Lewin AN, Zavanella C, Subramanian S. Sinus venosus atrial septal defect associated with partial anomalous pulmonary venous drainage: surgical repair. Ann Thorac Surg. 1978;26:185-8.

4. Warden HE, Gustafson RA, Tamay TJ, Neal WA. An alternative method for repair of partial anomalous pulmonary venous connection to the superior vena cava. Ann Thorac Surg. 1984;38:601-5.

5. Gaynor JW, Burch M, Dollery C, Sullivan ID, Deanfield JE, Elliott MJ. Repair of anomalous pulmonary venous connection to the superior vena cava. Ann Thorac Surg. 1995;59:1471-5.

6. Yamagishi M, Fujiwara K, Yaku H, Wada Y, Kitamura N. Repair of partial anomalous pulmonary venous connection with a minimal atriotomy. Jpn J Thorac Cardiovasc Surg. 2000;48:370-2.

7. Youssefi P, Sharma R, Figueroa CA, Jahangiri M. Functional assessment of thoracic aortic aneurysms-the future of risk prediction? Br Med Bull. 2017; 121:61-71.

8. Healey JE Jr. An anatomic survey of anomalous pulmonary veins: their clinical significance. J Thorac Surg. 1952;23:433-44.

9. Garduno C, Chew S, Forbess J, Smith PK, Grocott HP. Persistent left superior vena cava and partial anomalous pulmonary venous connection: incidental diagnosis by transesophageal echocardiography during coronary artery bypass surgery. J Am Soc Echocardiogr. 1999;12:682.

10. Lewis FJ. High defects of the atrial septum. J Thorac Surg. 1958;36:1-11.

11. Cooley DA, Latson JR, Keats AS. Surgical considerations in repair of ventricular and atrial septal defects utilizing cardiopulmonary bypass; experience with 104 cases. Surgery. 1958;43:214-25.

12. Ehrenhaft JL, Theilen EO, Lawrence MS. The surgical treatment of partial and total anomalous pulmonary venous connections. Ann Surg. 1957;148:249-58.

13. Anderson KR, Yen Ho S, Anderson RH. Location and vascular supply of sinus node in human heart. Br Heart J. 1979;41:28-32.

14. Ohmi M, Mohri H. A single pericardial patch technique for repair of partial anomalous pulmonary venous drainage associated with sinus venosus atrial septal defect. Ann Thorac Surg. 1988;46:360-1.

15. Kouchoukos N, Blackstone E, Doty D, Hanley F, Karp R. Kirklin/Barratt-Boyes Cardiac Surgery. 3rd ed. Philadelphia: Churchill Livingstone; 2003. 715-52.

16. Backer CL, Mavroudis C. Paediatric Cardiac Surgery. 3rd ed. Philadelphia: Mosby; 2003. 283-97.

17. Said SM, Burkhart HM, Schaff HV, Cetta F Jr, Phillips SD, Barnes RD, et al. Single-patch, 2-patch, and caval division techniques for repair of partial anomalous pulmonary venous connections: does it matter? J Thorac Cardiovasc Surg. 2012; 143:896-903.

18. Adachi I, Yagihara T, Kagisaki K, Hagino I, Ishizaka T, Koh M, et al. Fontan operation with a viable and growing conduit using pedicled autologous 
pericardial roll: serial changes in conduit geometry. J Thorac Cardiovasc Surg. 2005; 130:1517-22.

19. Guyton RA, Dorsey LM, Silberman MS, Hawkins HK, Williams WH, Hatcher JC. The broadly based pericardial flap. A tissue for atrial wall replacement that grows. J Thorac Cardiovasc Surg. 1984;87:619-25.

20. Stankovic Z, Allen BD, Garcia J, Jarvis KB, Markl M. 4D flow imaging with MRI. Cardiovasc Diagn Ther. 2014;4:173-92.

21. Ebbers T. Flow imaging: cardiac applications of 3D cine phase-contrast MRI. Curr Cardiovasc Imaging Rep. 2011;4:127-33.
22. Itatani K, Miyazaki S, Furusawa T, Numata S, Yamazaki S, Morimoto K, et al New imaging tools in cardiovascular medicine: computational fluid dynamics and 4D flow MRI. Gen Thorac Cardiovasc Surg. 2017;65:611-21.

23. Miyazaki S, Itatani K, Furusawa T, Nishino T, Sugiyama M, Takehara Y, et al Validation of numerical simulation methods in aortic arch using 4D flow MRI. Heart Vessels. 2017;32:1032-44.

Key Words: partial anomalous pulmonary venous return, intra-atrial rerouting, supraventricular arrhythmia 